\title{
STRUCTURAL, ELECTRONIC AND MAGNETIC PROPERTIES OF XYZ TYPE HALF-HEUSLER ALLOYS
}

\section{Rajendra Dahal and Gopi Chandra Kaphle}

Journal of Nepal Physical Society

Volume 5, Issue 1, October 2019

ISSN: $2392-473 X$

Editors:

Dr. Vinaya Kumar Jha

Dr. Binod Adhikari

Dr. Kapil Adhikari

JNPS, 5 (1), 97-102 (2019)

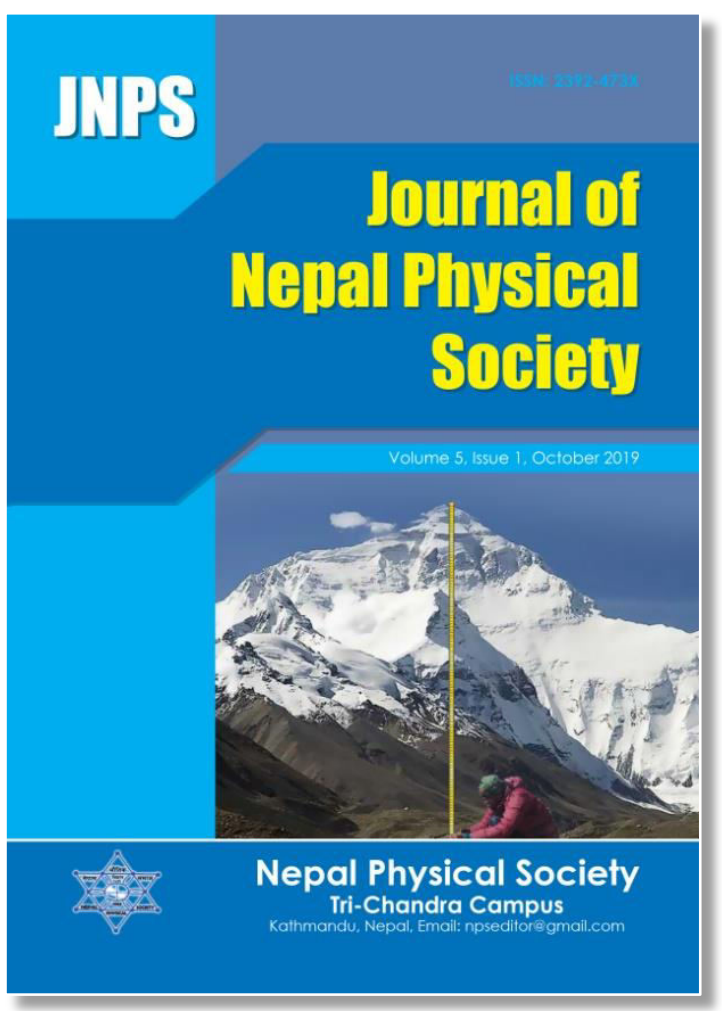

Published by:

Nepal Physical Society

P.O. Box: 2934

Tri-Chandra Campus

Kathmandu, Nepal

Email:npseditor@gmail.com 


\title{
STRUCTURAL, ELECTRONIC AND MAGNETIC PROPERTIES OF XYZ TYPE HALF-HEUSLER ALLOYS
}

\author{
R. Dahal ${ }^{1}$, and G. C. Kaphle ${ }^{2,3, *}$ \\ ${ }^{I}$ Departement of Physics, Amrit Science Campus, Kathmandu, Nepal \\ ${ }^{2}$ Hydra Research and Policy Center, Kathmandu, Nepal \\ ${ }^{3}$ Central Department of Physics, Tribhuvan University, Kirtipur, Kathmandu, Nepal \\ *Corresponding Email: gck223@gmail.com
}

\begin{abstract}
The spintronic devices have played an important role in modern technological era . Heusler alloys have attracted lot of interest in spintronic applications due to their half-metallic properties predicted by band structure calculations. We investigate the electronic, magnetic and structural properties of half-Heusler alloys FeMnGe and CoMnSb using first principles based density functional theory (DFT) implemented on Tight Binding Linear Muffin-Tin Orbital within Atomic Sphere Approximation (TB-LMTO-ASA) code. The calculation reveal that $\mathrm{CoMnSb}$ and FeMnGe are half-metallic Ferro-magnet in nature of with magnetic moment $1.00 \mu \mathrm{B}$ and $2.99 \mu \mathrm{B}$ per formula unit at equilibrium lattice parameter respectively. The magnetic moment mainly originates from the strong spin polarization of $\mathrm{d}$ electrons of $\mathrm{X}$ atom and partial contribution of $\mathrm{p}$ electrons of $\mathrm{Y}$ atom. The half metallic gap of FeMnGe and $\mathrm{CoMnSb}$ is found to be $0.38 \mathrm{eV}$ and $0.95 \mathrm{eV}$ respectively. This shows that these alloys are very promising spintronic functional materials.
\end{abstract}

Keywords: TB-LMTO-ASA, half-Heusler Alloy, half-Metallicity, DOS, Spintronic applications

\section{INTRODUCTION}

The spintronic devices have played an important role in modern technological era. It is also very important for fundamental aspect of Physics. Half-metallic (HM) materials are those one which one of the spin bands out of two, is semiconducting with a gap at the Fermi level, but another spin band is metallic, leading to $100 \%$ spin polarization at the Fermi level, have attracted more and more attention because of their promising applications in spintronic devices. First de Groot et al. [1] predicted the half metallic property in half-Heusler alloy of $\mathrm{NiMnSb}$ and $\mathrm{PtMnSb}$, since then much attention has been paid to half-Heusler alloys [2]. The term Heusler alloy is named after a German mining engineer and chemist Friedrich Heusler in 1903 [3, 4]. Surface reconstruction has been an active area of research in field of semiconductors. The basic thing of the electronic devices is to inject the spin polarized electrical current into semiconductors [5]. In Ferromagnet the spin population is not balance at Fermi level. Hence ferromagnetic materials with full spin polarization at Fermi level will be the most applicable for spin injecting. This phenomenon can be applicable in half metallic Ferromagnet. HalfHeusler composition of XYZ, where $\mathrm{X}$ and $\mathrm{Y}$ are transition metal elements, $\mathrm{Z}$ is a main group element. In recent year, Heusler compounds containing Co and $\mathrm{Mn}$ atoms have attracted particular attention as they are strongly ferromagnetic with high Curie temperature [6]. The half-Heusler alloy is trust worthy materials to be applied as spin injector in the rapidly developing field of spin electronics (spintronic) [7]. The rapidly developing field of electronics technology is owing to the discoveries of GMR and TMR which opened the line of research in spintronic [8, 9]. In half metallic Ferro magnet (HMFs) the majority of the spin band is metallic and the minority of the spin band is superconducting with an energy gap at the Fermi level. DOS of half metals compared with metals and semiconductors is shown in Fig. (1).

From figure 1 above it is lucid that there is no band gap in the Fermi region for metals but in case of semiconductors there is gap. Half metals have no band gap in spin up channel at the Fermi level but has considerable band gap in the spin down channel. This shows that spin up electrons of Fermi level shows metallic nature while spin down electrons Fermi level shows non-metallic nature. Combining these two parts we can conclude that system is half metallic. Thus Heusler alloy are 
promising material for spin devices. The existence of a gap in the minority spin band structure leads to $100 \%$ spin polarization of the electron states at Fermi level, which makes the system applicable for the developing field of spintronic [10].
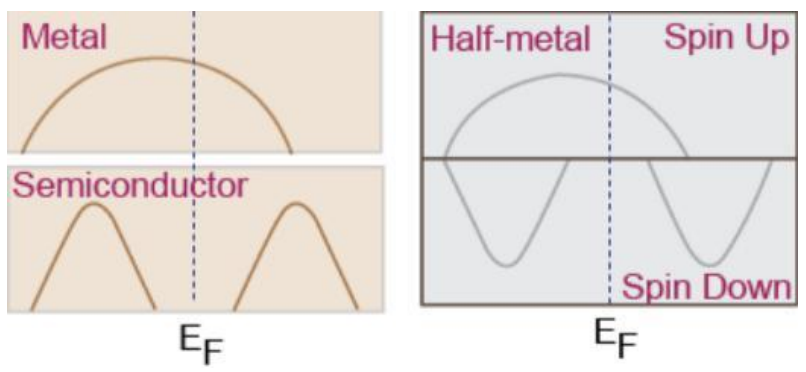

Fig.1: (color online) Density of states of metals, semiconductors \& half-metals.

Therefore these materials utilize the spin in addition to the charge of electron to carry the current. HMFs have completely spin polarised current and can be used as spin injector for magnetic random access memories and other spin dependent devices [11]. We can considered half metals as hybrids metals and semiconductors. The spin polarization makes this alloy prime target research into suitable devices for spin electronics. As these types of alloys are most attractive half metals, both electronic and magnetic properties in these compounds are intrinsically related to the appearance of minorityspin gap thus opening the way to engineering new half metallic alloys with the desired magnetic property [12]. For both scientific and technological reasons it is important to determine the electron spin polarization at the Fermi level of a material, although it is difficult to measure [13].

$$
\mathbf{P}=\frac{N \uparrow(E f)-N \downarrow(E f)}{N \uparrow(E f)+N \downarrow(E f)}
$$

Where $\mathrm{N} \uparrow(\mathrm{Ef})$ and $\mathrm{N} \downarrow(\mathrm{Ef})$ are the spin dependent density of states at the fermi level. When

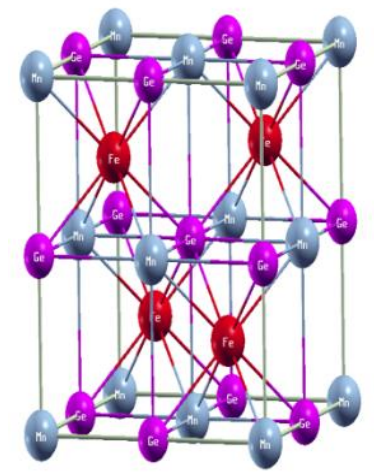

Fig. 2: (color online) Crystal structure of FeMnGe and CoMnSb for unit cell. the electrons are fully spin polarized, either $\mathrm{N} \uparrow($ Ef) or $\mathrm{N} \downarrow(\mathrm{Ef})$ equal zero and therefore the spin polarization will be $100 \%$ [14, 15] A systematic examination of the electronic and magnetic structure of the half-Heusler compounds was carried out this work. The Slater-Pauling rule was first noted by Kubler et al. [16] for C1b compounds with 3 atoms per unit cell ( $\mathrm{Mt}=\mathrm{Nv}-18)$. In the present study, we are intended to apply TBLMTOASA approach, which was previously applied in case of ordered binary alloys, ternary alloys, disordered alloys and perovskites[17-23].

\section{CRYSTAL STRUCTURE AND METHODS}

In general, the Heusler structure can be looked on as four interpenetrating face center cubic (FCC) lattice and has four unique crystal-sites in Wyckoff coordinates [24]. FeMnGe and CoMnSb crystallize in the $\mathrm{C} 1 \mathrm{~b}$ structure which is face-centered cubic, space group F-43m (Space group no. 216). This structure type is often observed for ternary transition-meal intermetallic compounds (XYZ). Actually it is like ordinary L21 like full Heusler alloys (X2YZ) with fcc lattices characterized as the positions X1(1/4 1/4 1/4), X2 (3/4, 3/4 , 3/4), Y (0, $0,0)$, and $Z(1 \backslash 2,1 \backslash 2,1 \backslash 2)$ with the exception that the $\mathrm{X} 1$ positions are empty. The nearest-neighbor coordination of the $\mathrm{X} 2$ atoms is similar in the two types of Heusler alloys X2YZ and XYZ. This Coordination consists of two interpenetrating tetrahedrons, involving four $\mathrm{Y}$ atoms and four $\mathrm{z}$ atoms, respectively. Some groups $[25,26]$ have verified that site preference of the $\mathrm{X}$ and $\mathrm{Y}$ atoms is strongly influenced by the number of their $3 d$ electrons in the complicated crystallization process. The half-Heusler alloy FeMnGe and CoMnSb (both $\mathrm{Clb}$ structure, space group: F-43m and space group no. 216 can be deduced from the full-Heusler $\mathrm{Fe} 2 \mathrm{MnGe}$ and $\mathrm{Co} 2 \mathrm{MnSb}$ (L21 structure, space group: $\mathrm{Fm}-3 \mathrm{~m}$ ) by removing one of two $\mathrm{Fe}$ and $\mathrm{Co}$ atoms respectively.

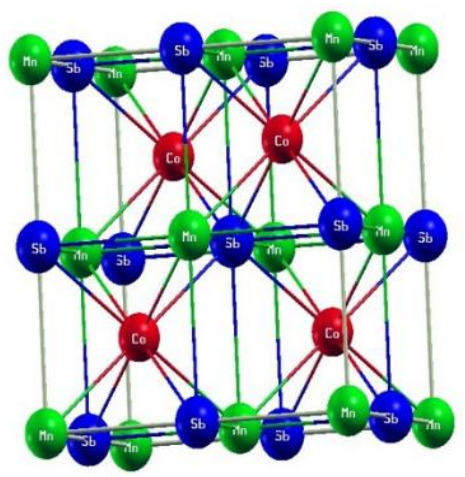


For the calculation we have used the TBLMTOASA method based on the density functional theory (DFT) [27, 28]. We have optimized the structure of $\mathrm{CoMnSb}$ and $\mathrm{FeMnGe}$ to get the energy minimization; through which we obtained the lattice parameters $5.72 \mathrm{~A}^{0}$ and $5.46 \mathrm{~A}^{0}$ respectively. These values of lattice parameter are closely agreed with the experimental values $[26,29]$ and the further calculations have been carried out with the use of these values. The energy convergence criterion was set to $10^{-6}$ Ryd.

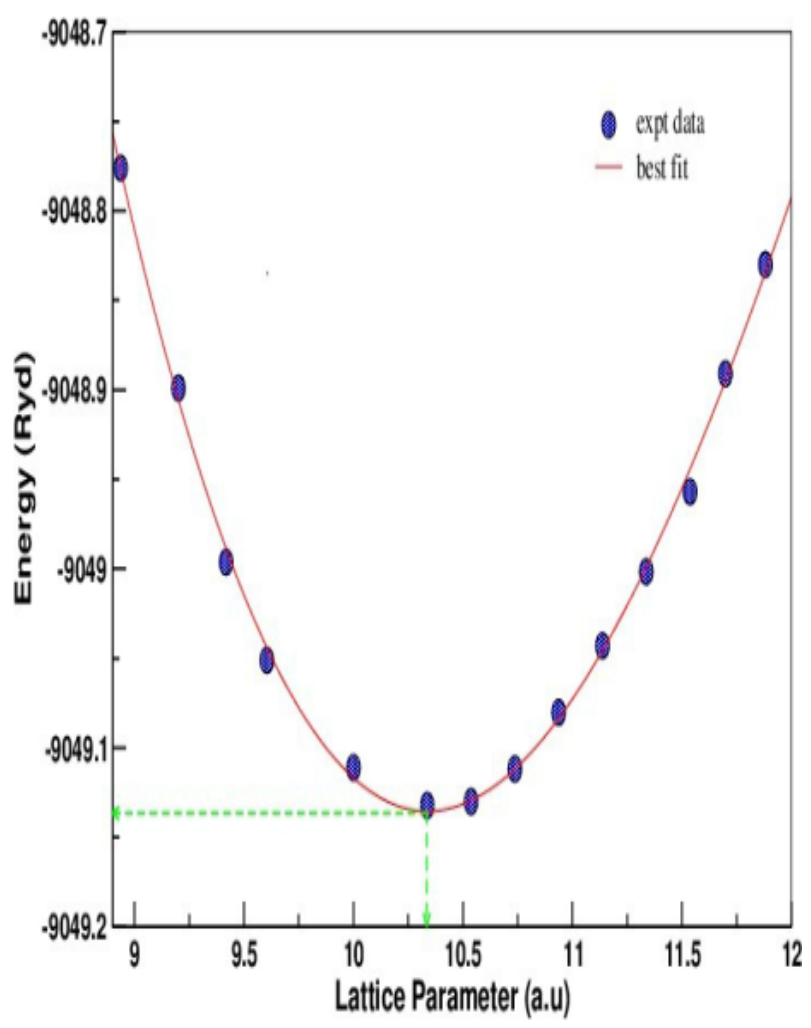

\section{RESULTS AND DISCUSSION}

\section{A. Band Structure and Dos}

To determine the equilibrium lattice parameter value, we follow the energy minimization procedure. The simple figure showing variation of total energy with the lattice parameters for $\mathrm{CoMnSb}$ and FeMnGe are shown in figure 3. From the figure the optimized value of lattice parameter for FeMnGe and $\mathrm{CoMnSb}$ are found to be $5.46 \mathrm{~A}^{\circ}$ and $5.72 \mathrm{~A}^{\circ}$ respectively. These parameters are now used to calculate the band structure and density of states as well as other required calculations.

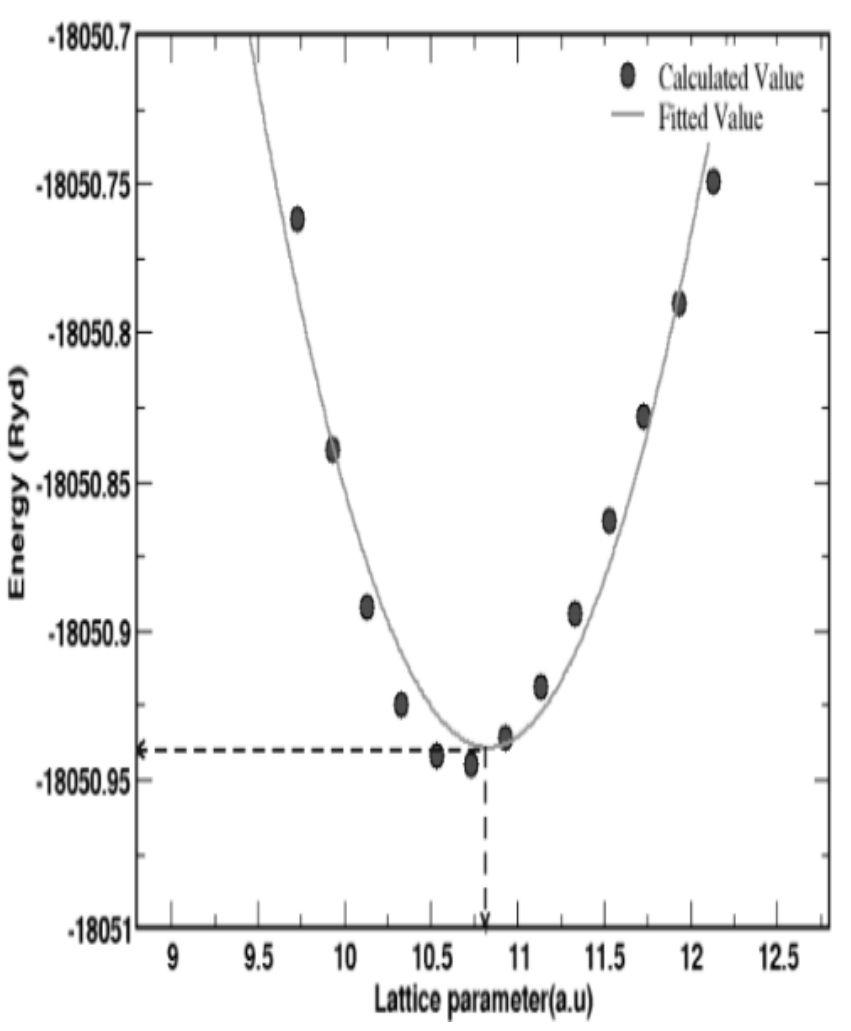

Fig. 3: (color online) Plot of energy Vs Lattice constant for FeMnGe and CoMnSb.

From the calculation of band structure of FeMnGe and $\mathrm{CoMnSb}$ for the majority of spin channel found to be crossed the fermi level indicating that up spin channel is metallic in nature same kinds of behavior can be observed in the density of states curve. The combined figures of band and DOS for FeMnGe and $\mathrm{CoMnSb}$ are shown in figure (4). The nature of DOS exactly follows the results given by band. In case of minority channel band structure calculations for $\mathrm{FeMnGe}$ and $\mathrm{CoMnSb}$ are found to be considerable band gaps i. e. $0.33 \mathrm{eV}$ and $0.95 \mathrm{eV}$. The main contributions of band gap comes from the hybridization of eg and $\mathrm{t} 2 \mathrm{~g}$ orbitals of the $\mathrm{Fe}, \mathrm{Mn}$ and and P- orbital of Ge in case of FeMnGe where as in case of $\mathrm{CoMnSb}$, the hybridization of eg and t2 $\mathrm{g}$ orbitals of $\mathrm{Co}, \mathrm{Mn}$ and P-orbital of the $\mathrm{Sb}$. The contribution of eg and $\mathrm{t} 2 \mathrm{~g}$-orbitals of $\mathrm{Fe} / \mathrm{Co}$ and $\mathrm{Mn}$ is may be due to the de-localized electrons. Overall we observed 21 bands inter-playing with each other to give rise to metallic as well as semiconducting behavior on the up-spin and downspin channel. The contributions of $s, p$ and $d$ orbitals can be visualized using fat band calculations and partial density of states. 

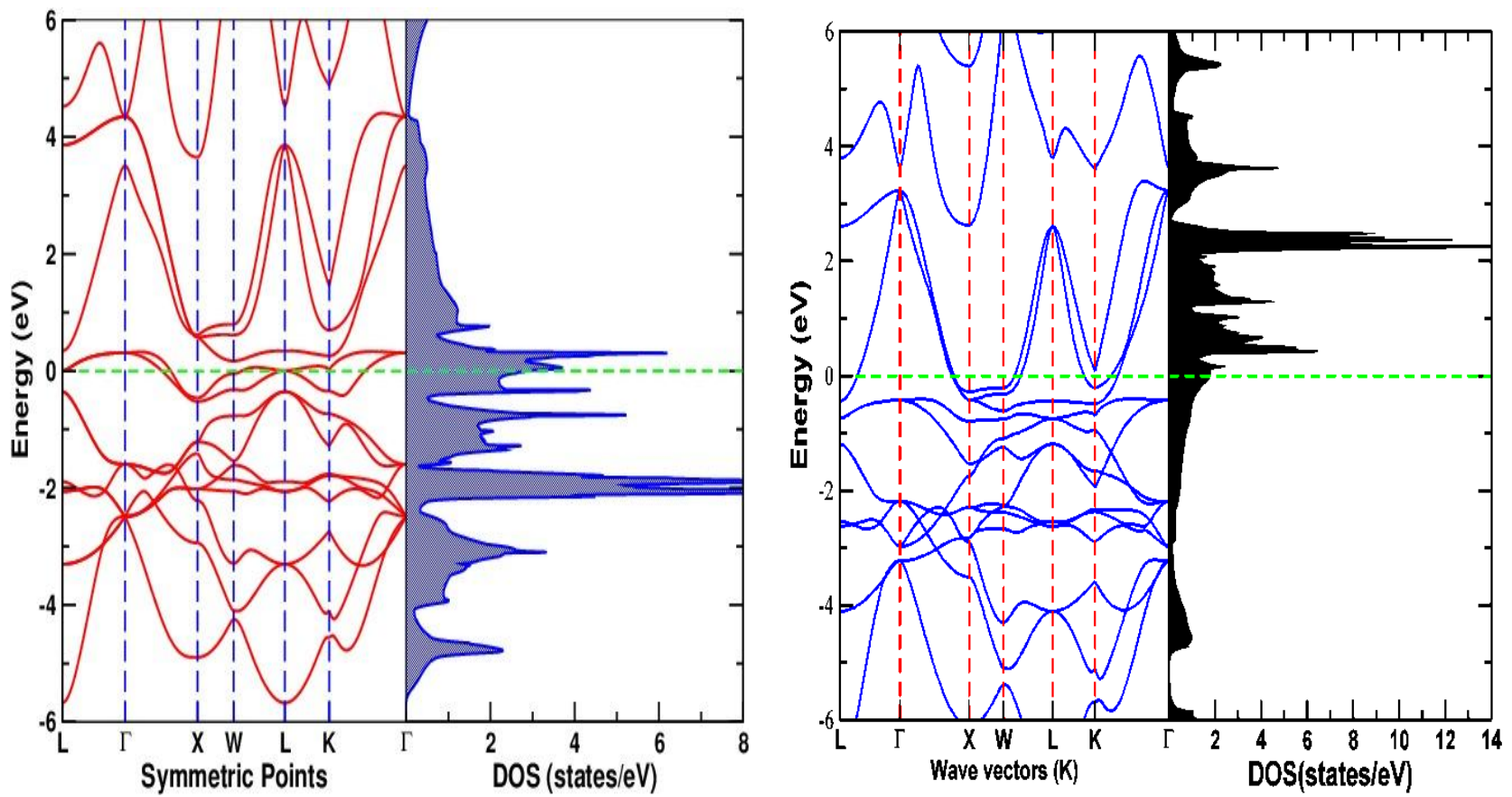

Fig. 4: (color online) Comparison of majority-spin band with the majority-spin DOS for FeMnGe \& CoMnSb.
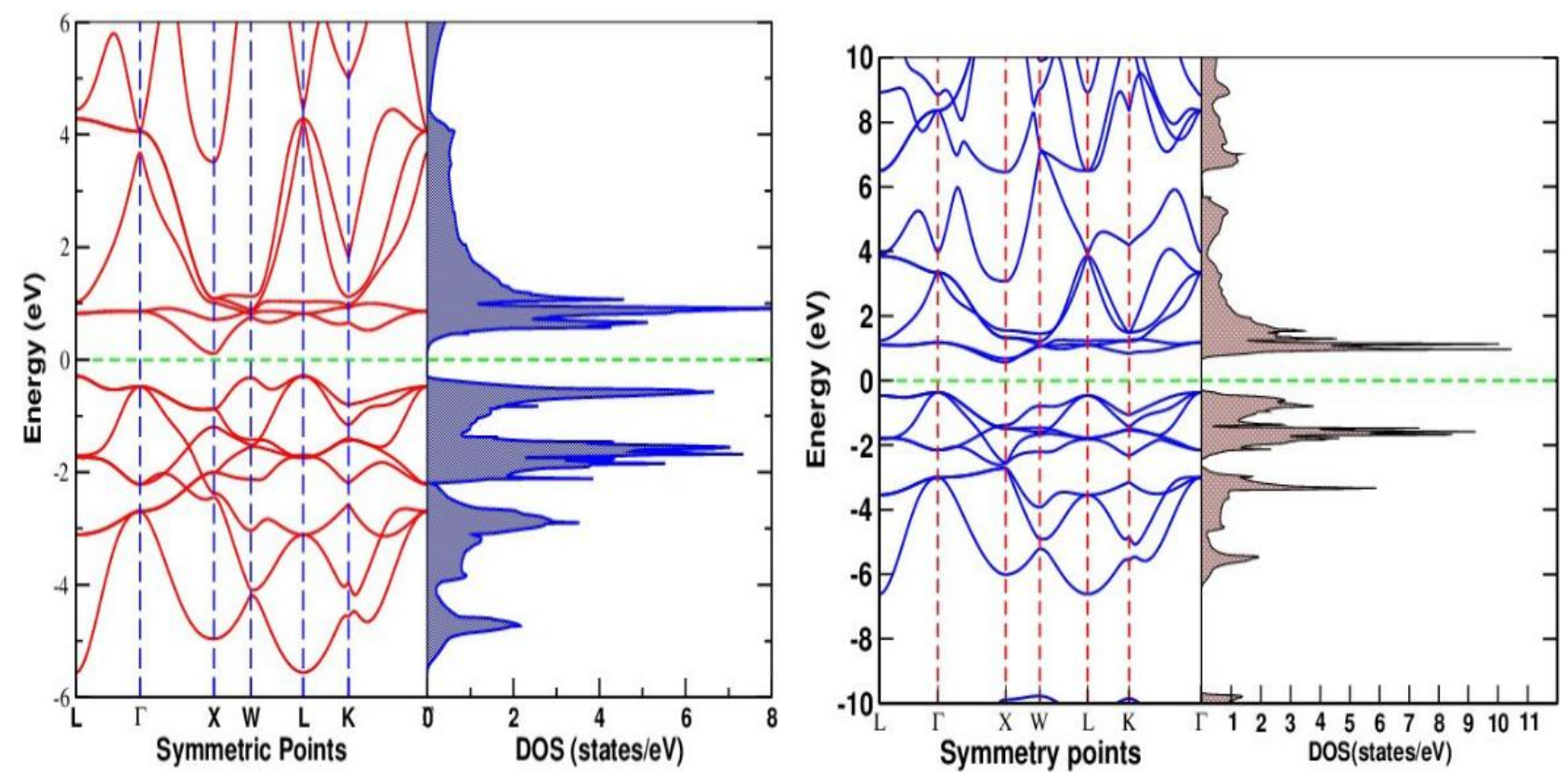

Fig. 5: (color online) Comparison of minority-spin band with the minority-spin DOS for FeMnGe \& CoMnSb.

The combined structures of up spin and down spin channel total dos is shown in figure (6). From the figure it is observed that there is unsymmetrical distributions of DOS on up spin and down spin channels on both the systems indicating that both bears magnetic character. Again analysis of DOS shows that both the systems have $100 \%$ spinpolarization at the Fermi level. 

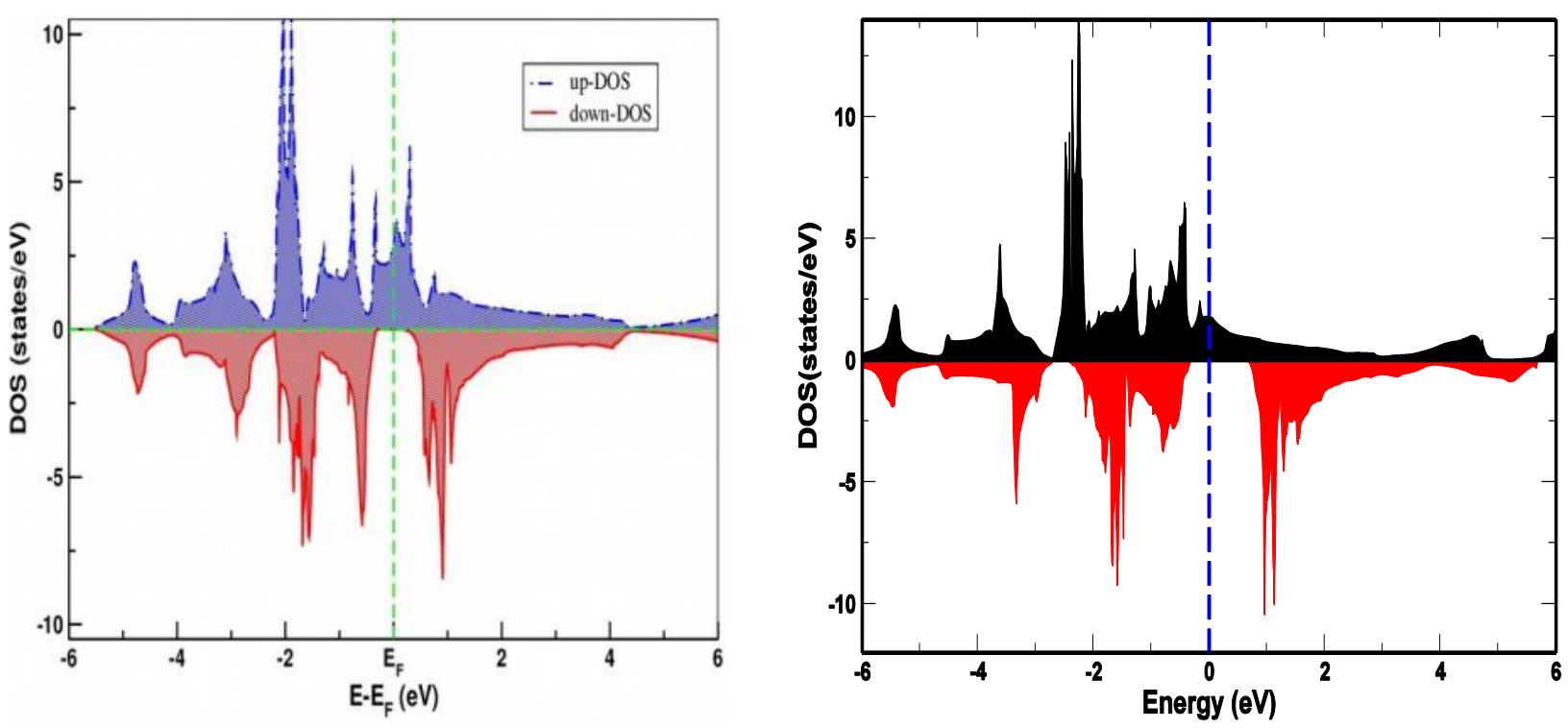

Fig. 6: (color online) Total DOS of FeMnGe and CoMnSb.

The alternative $\mathrm{Y}$-axis parallel to $\mathrm{X}$-axis represents the Fermi level Ef and above the alternative $\mathrm{X}$-axis represents the majority-spin DOS and below the alternative $\mathrm{X}$-axis represents the minority-spin DOS. This also supports the half metalic characters of FeMnGe and $\mathrm{CoMnSb}$ with half metalic gap $0.33 \mathrm{eV}$ and $0.95 \mathrm{eV}$ as previously defined by band.

\section{B. Magnetic Moments}

The unsymmetrical nature of DOS on up and down spin and charge density distributions from electronic properties of $\mathrm{FeMnGe}$ and $\mathrm{CoMnSb}$ give rise to the magnetic properties of these systems. This magnetic moments mainly originates from the t2 $\mathrm{g}$ and eg d-orbitals of $\mathrm{Fe}$ and Co hybridized with $p$ orbitals. The individual contributions of magnetic moments are listed in the Table 1 below.

Table 1: Contributions of magnetic moments.

\begin{tabular}{lccccc}
\hline Compound & $\mathbf{C o \& F e}\left(\boldsymbol{\mu}_{\mathbf{B}}\right)$ & $\mathbf{M n \& F e}\left(\boldsymbol{\mu}_{\mathbf{B}}\right)$ & $\mathbf{S b \& ~ G e}\left(\boldsymbol{\mu}_{\mathrm{B}}\right)$ & $\mathbf{m}^{\text {Tot }}\left(\boldsymbol{\mu}_{\mathbf{B}}\right)$ & $\mathbf{E x p .}\left(\boldsymbol{\mu}_{\mathrm{B}}\right)$ \\
\hline $\mathrm{CoMnSb}$ & -0.070 & 3.170 & -0.110 & 2.990 & $3[30]$ \\
$\mathrm{FeMnGe}$ & -0.863 & 1.912 & -0.048 & 1.001 & $1[31]$ \\
\hline
\end{tabular}

Present calculations fairly agree with the experimental values and $\mathrm{CoMnSb}$ found to be strong ferromagnetic whereas FeMnGe is weak ferromagnetic in nature.

\section{CONCLUSIONS}

In the present calculation, we studied the crystal structure, band structure, density of states and magnetic moment of Heusler alloys like FeMnGe and CoMnSb using TB-LMTO-ASA method within the framework of Density Functional Theory (DFT). At first the value of lattice parameter corresponding to the minimum energy has been calculated through energy minimization process. The lattice parameter corresponding to minimum energy for Heusler alloys FeMnGe and $\mathrm{CoMnSb}$ are found to be within the $0.18 \%$ and $2.63 \%$ errorbar compared to the experimental values i. e. $5.46 \mathrm{~A}^{\circ}$ and $5.72 \mathrm{~A}^{\circ}$ respectively. We calculated the electronic and magnetic properties of using optimized value of lattice parameter. In halfHeusler Alloys overlapping bands were found for majority spin channel while certain band gap of $0.38 \mathrm{eV}$ for $\mathrm{FeMnGe}$ and $0.95 \mathrm{eV}$ for $\mathrm{CoMnSb}$ were found for minority spin channel indicating that present Heusler alloys are half-metallic in nature. To know the individual contribution on band and DOS we used fat band calculation. From the fat band calculations it is found that the main contributions of bands in the prescribed systems are 
mainly came from d-orbitals of $\mathrm{Fe}$ (or $\mathrm{Co}$ ) and $\mathrm{Mn}$ and p-orbital of $\mathrm{Ge}($ or $\mathrm{Sb}$ ). From the study of density of states,Heusler alloys showed magnetic nature which is indicated by asymmetric nature of up and down DOS along the Fermi level. The magnetic moment of $\mathrm{FeMnGe}$ and $\mathrm{CoMnSb}$ is found to be $1.00 \mu \mathrm{B}$ and $2.99 \mu \mathrm{B}$ respectively.

\section{ACKNOWLEDGMENTS}

This work is supported partially by HRCBS, Kathmandu, Nepal. N. P. Adhikari of Central Department of Physics, Tribhuvan University is acknowledged for suggestions during the preparation of manuscript and computational code for the project.

\section{REFERENCES}

[1] R. A. de Groot and F. M. Muller, Physical review letters, 50 (25), 2024 (1983).

[2] J. Pierre, R. V. Skolozdra, J. Tobola, S. Kaprzyk, C. Hordequin, M. A. Kouacou, I. Karla, R. Currat and E. Leli, J. Alloys Comp. 101, 262 (1997).

[3] B. Hamad, J. Khalifeh and H. Luo, The electronic structure and spin polarization of Alloys, J. Appl. Phys. 107, 903 (2010).

[4] I. Shigeta, S. Urakawa, M. Ito and M. Hiroi, Magnetization and spin polarization of Heusler alloys, J. Phys. 400, 032082 (2012).

[5] W. van Roy, de Boeck, J. Das, V. Motsnyi, Z. Liu, L. Lagae, H. Boeve, K. Dessein and G. Borghs, Semicond. Sci. Technol. 17, 342 (2002).

[6] S. E. Kulkova, S. V. Eremeev, and S. S. Kulkov, Solid State Communications , 130 (12), 793 (2004). [7]U. V. Barth, Phy. Scr., 9 (2004).

[8] S. J. Hashemifar, P. Kratzer and M. Scheffler PRL, 94, 1 (2005).

[9] J. De Boeck, W. Van Roy, J. Das, V. Motsnyi, Z. Liu, L. Lagae, H. Boeve, K. Dessein and G. Borghs Semicond. Sci. Technol. 17(4), 342 (2002).

[10] C. Felser, G. H. Fecher and B. Balk, Angew. Chem., Int. Ed. 46, 668 (2007).

[11] A. M. Boldin, Macalester Journal of Physics and Astronomy, 3(1), Art. 3 (2015)

[12] I. Galanakis, Ph. Mavropoulos and P. H. Dederichs, Journal of Physics D: Applied Physics, 39(5), 765 (2006).
[13] D. P. Rai, Sandeep, A. Shankar, A. P. Sakhya, T. P. Sinha, R. Khenata, M. P. Ghimire and R. K. Thapa, Mater. Res. Express, 3(7), 075022 (2016).

[14] H. C. Kandpal, G. H. Fecher, C. Felser, Journal of Physics D: Applied Physics, 40(6), 1507 ( 2007).

[15] S. Wurmehl, G. H. Fecher, H. C. Kandpal, V. Ksenofontov, C. Felser, H. Lin, J. Morais, Phys. Rev. B. 72(18), 184434 (2005).

[16] J. Kübler, A. R. William, and C. B. Sommers, Phys. Rev. B 28, 1745 (1983).

[17] S. Pandey, G. C. Kaphle, N. P. Adhikari, BIBECHANA 11, 60 (2014).

[18] S. Dahal, G. Kafle, G. C. Kaphle, N. P. Adhikari, Journal of Institute of Science and Technology 19 (1), 137 (2015).

[19] S. Lamichhane, G. C. Kaphle, N. P. Adhikari, Quantum Matter 5 (3), 356(2016).

[20] P. Pal, R. Banerjee, A. Mookerjee, G. C. Kaphle, B. Sanyal, J. Hellsvik, O. Eriksson, P. Mitra, A. K. Majumdar, A. K. Nigam Physical Review B 85 (17), 174405 (2012).

[21] G. C. Kaphle, S. Ganguly, R. Banerjee, R. Banerjee, R. Khanal, C. M. Adhikari, N. P. Adhikari, A. Mookerjee, Journal of Physics: Condensed Matter 24 (29), 295501 (2012).

[22] G. C. Kaphle, N. Adhikari, A. Mookerjee Advanced Science Letters 21 (9), 2681 (2015).

[23] S. Lamichhane, B. Aryal, G. C. Kaphle, N. P. Adhikari BIBECHANA 13, 94 (2014).

[24] http://www.cryst.ehu.es/cgibin/cryst/programs/nph-wp-list

[25] A. Ayuela, J. Enkovaara, K. Ullakkod and R. M. Nieminen, J. Phys.: Condens. Matter, 11(6), 2017 (1999).

[26] A. SzytuŁa, A. T. Pȩdziwiatr, Z. Tomkowicz, W. Bażela, J. Magn. Magn. Mater., 25(2), 176 (1981).

[27] O. K. Andersen, Phys. Rev. B 12, 3060 (1975).

[28] O. K. Andersen and O. Jepsen, Phys. Rev. Lett. 53, 2571 (1984).

[29] V. Ksenofontov, G. Melnyk, M. Wojcik, S. Wurmehl, K. Kroth, S. Reiman, P. Blaha, and C. Felser, Phys. Rev. B 74, 134426 (2006).

[30] C. H. Park, B. C. Lee and J. I. Lee, J. Korean Phys. Soc. 47(4), 655 (2005).

[31] L. Feng, E. K. Liu, W. X. Zhang, W. H. Wang, G. H. Wu, J. Magn. Magn. Mater. 351, 92 (2014). 